\title{
DEVELOPMENT OF A MACHINE-VISION-BASED SYSTEM FOR RECORDING OF FORCE CALIBRATION DATA
}

\author{
SUMET HEAMAWATANACHAI \\ Department of Mechanical Engineering, Naresuan University \\ Phitsanulok 65000, Thailand \\ sumeth@nu.ac.th, sumet@nimt.or.th \\ KITTIPONG CHAEMTHET \\ National Institute of Metrology (Thailand) \\ Pathum Thani 12120, Thailand \\ kittipong@nimt.or.th \\ TAWAT CHANGPAN \\ National Institute of Metrology (Thailand) \\ Pathum Thani 12120, Thailand \\ tawat@nimt.or.th
}

\begin{abstract}
This paper presents the development of a new system for recording of force calibration data using machine vision technology. Real time camera and computer system were used to capture images of the reading from the instruments during calibration. Then, the measurement images were transformed and translated to numerical data using optical character recognition (OCR) technique. These numerical data along with raw images were automatically saved to memories as the calibration database files.

With this new system, the human error of recording would be eliminated. The verification experiments were done by using this system for recording the measurement results from an amplifier (DMP 40) with load cell (HBM-Z30-10kN). The NIMT's 100-kN deadweight force standard machine (DWM-100kN) was used to generate test forces. The experiments setup were done in 3 categories; 1) dynamics condition (record during load changing), 2) statics condition (record during fix load), and 3) full calibration experiments in accordance with ISO 376:2011.

The captured images from dynamics condition experiment gave $>94 \%$ without overlapping of number. The results from statics condition experiment were $>98 \%$ images without overlapping. All measurement images without overlapping were translated to number by the developed program with $100 \%$ accuracy. The full calibration experiments also gave $100 \%$ accurate results. Moreover, in case of incorrect translation of any result, it is also possible to trace back to the raw calibration image to check and correct it. Therefore, this machine-vision-based system and program should be appropriate for recording of force calibration data.
\end{abstract}

Keywords: Force measurement; calibration; machine vision; OCR; ISO 376.

This is an Open Access article published by World Scientific Publishing Company. It is distributed under the terms of the Creative Commons Attribution 3.0 (CC-BY) License. Further distribution of this work is permitted, provided the original work is properly cited. 


\section{Introduction}

In the field of force measurement, load cell is an instrument for measuring force in many material testing applications, ranging from small force as testing food products to large force as testing properties of steel for construction. Generally, the calibrations of a reference load cells are carried out according to standard procedure such as ISO $376^{[1]}$. The calibration procedure of this standard is shown in Fig. 1.

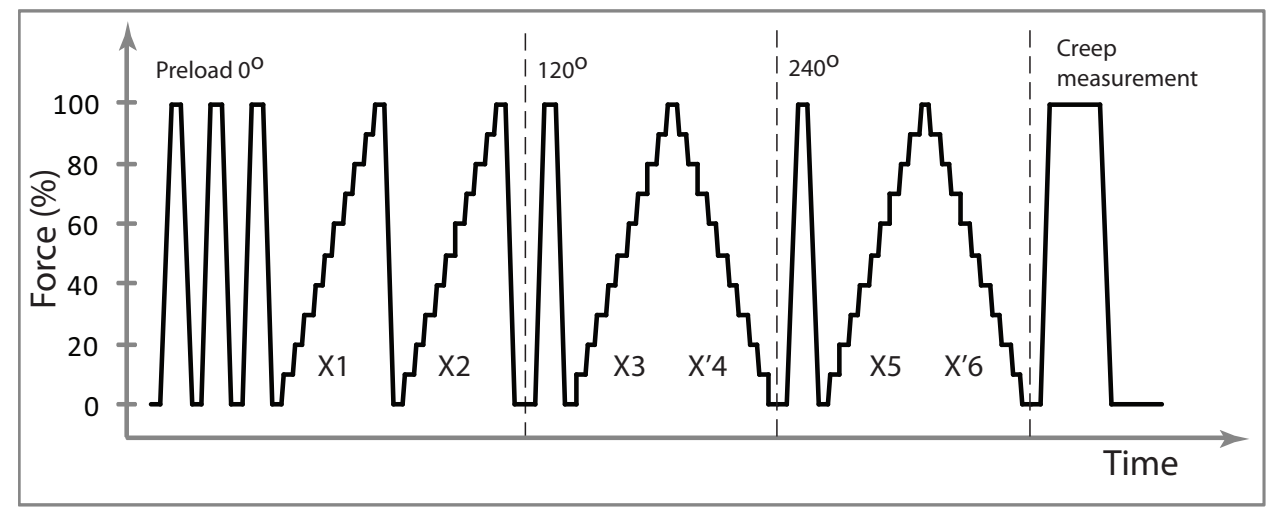

Fig.1. Calibration procedure according to ISO 376:2011.

As shown in Fig. 1, each calibration contains of 6 series; $\mathrm{x} 1$ and $\mathrm{x} 2$ are increasing series at $0^{\circ}$ angle, $\mathrm{x} 3$ and $\mathrm{x}^{\prime} 4$ are increasing and decreasing series at $120^{\circ}$ angle, $\mathrm{x} 5$ and x' 6 are increasing and decreasing series at $240^{\circ}$ angle. The total calibration period is about 3 to 5 hours, which is depend on type of the calibration machine and load cell. Generally, for force calibration at the National Institute of Metrology Thailand (NIMT) (see Fig. 2(a)), the operator manually write down the reading of the force proving instrument into a calibration sheet. Then, input all the written data into the calibration file for calculation. After issuing a certificate, the supervisor will check the correctness of the calibration certificate before signing into the certificate for approving.

From experiences, there were many human errors from manually recording the calibration data. In case of mistakes with the input data, the operator could re-input the data and re-issue a calibration certificate. In case of mistakes with the written data in calibration sheet, it is nearly impossible to trace back to find the source of the problem from the regular method of recording data. Therefore, re-calibration was necessary to ensure the correctness of the calibration results.

From previous studies, automatic recording data systems were developed and achieved by using communication port such as RS232 and GPIB to connect to the measuring instruments. However, there are no communication ports for some instruments, and various kinds of communication protocols from many different manufacturers, it is not practical to apply this method for most calibrations. 


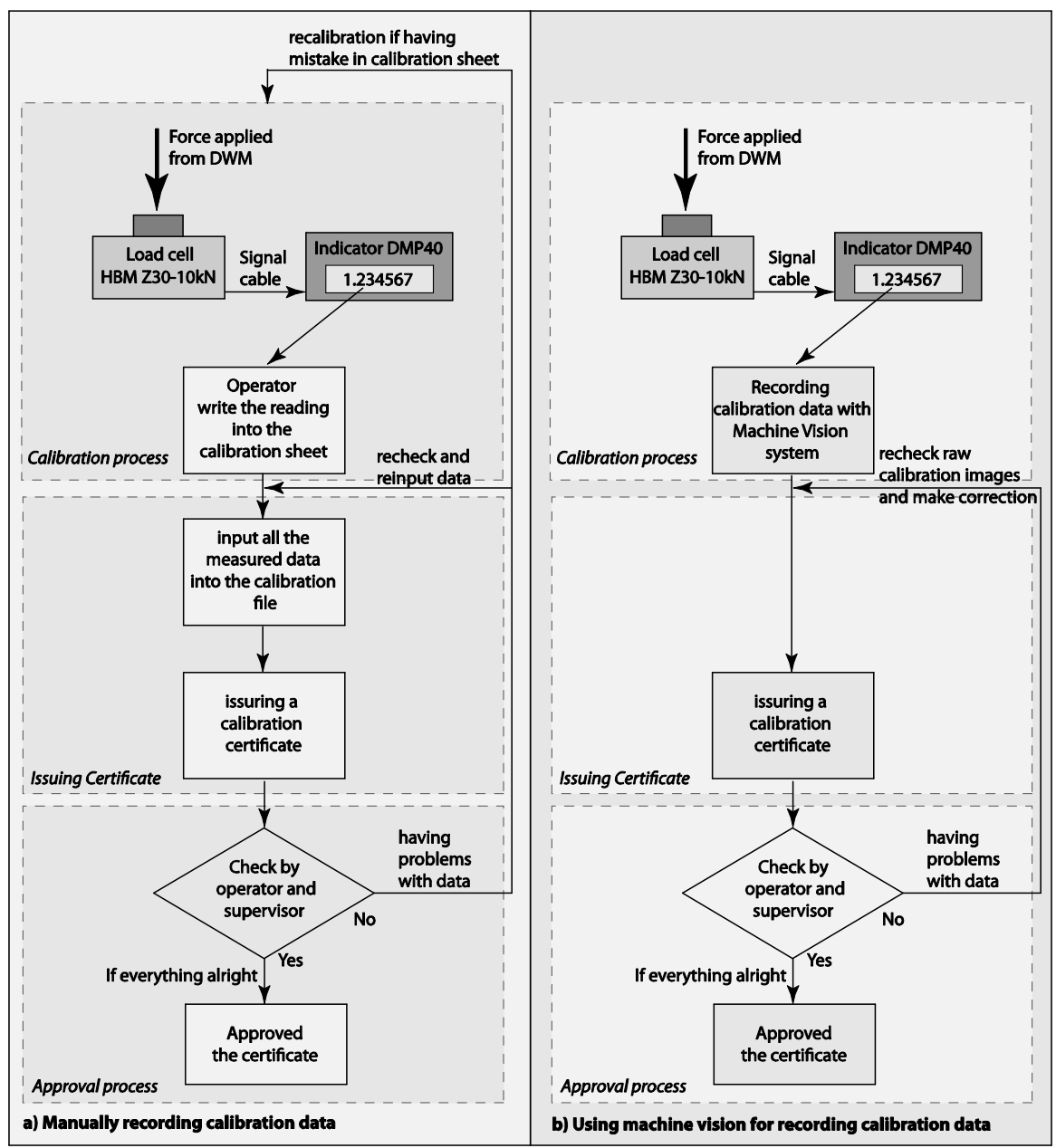

Fig.2. Force calibration workflow.

The current problems on reading and recording ability of the operator could be eliminated by using Machine vision technology, which is a powerful technique that allows computer to understand the image of objects and make decision base on the condition of that image for controlling. This technology was used in many applications such as reading bar code and face detection. Wang et al. used machine vision technique for inspection of surgical instruments ${ }^{[2]}$. Sun et al. identified defects on electrical contact using machine vision ${ }^{[3]}$. Others applications such as detection of fabric defects ${ }^{[4]}$ and electronics parts ${ }^{[5]}$ are also available.

Optical character recognition (OCR) is a technique of machine vision technology. This technique allows machine or computer to recognize image of characters. It is the electronic translation of images of handwritten, typewritten or printed text into machineeditable text ${ }^{[6]}$. This technique can be used for calibration of digital instrument ${ }^{[7]}$. 
As seen in Fig. 2(b), a new system and program using machine vision technique was developed for recording of force calibration data. During calibration, the image of reading form instruments will be recorded with a real time camera system. Then, the image will be processed with some machine vision techniques ${ }^{[8,9]}$ such as setting region of interest (ROI), segmentation, transformation, etc. After that, the processed image will be analyzed to obtain numerical values of measurement images using the optical character recognition technique (OCR) as seen in Fig. 3. These images, measuring values along with measuring times will be automatically saved to the calibration database files for later calculation.

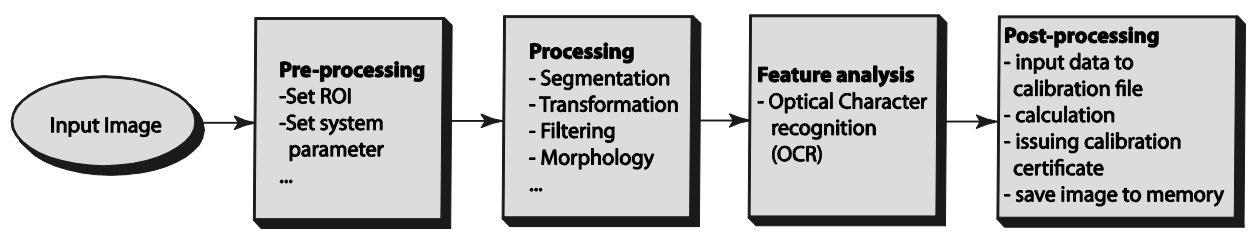

Fig.3. Image analysis process.

\section{Experimental System Setup}

As shown in Fig. 4, the developed machine vision system composed of a GigE camera and a developed program. In order to verify the system and to prove that this technique is appropriate for force calibration. A 100-kN Deadweight Force Standard Machine (DWMMaekawa-FSD-10) was used for force generation (Fig. 4). Load cell (HBM-Z30-10kN) with amplifier (HBM-DMP40) were used for measuring the applied forces. The developed machine vision system was used to capture image of measurement result from the amplifier (HBM-DMP40) and translate the measurement image to numerical data.

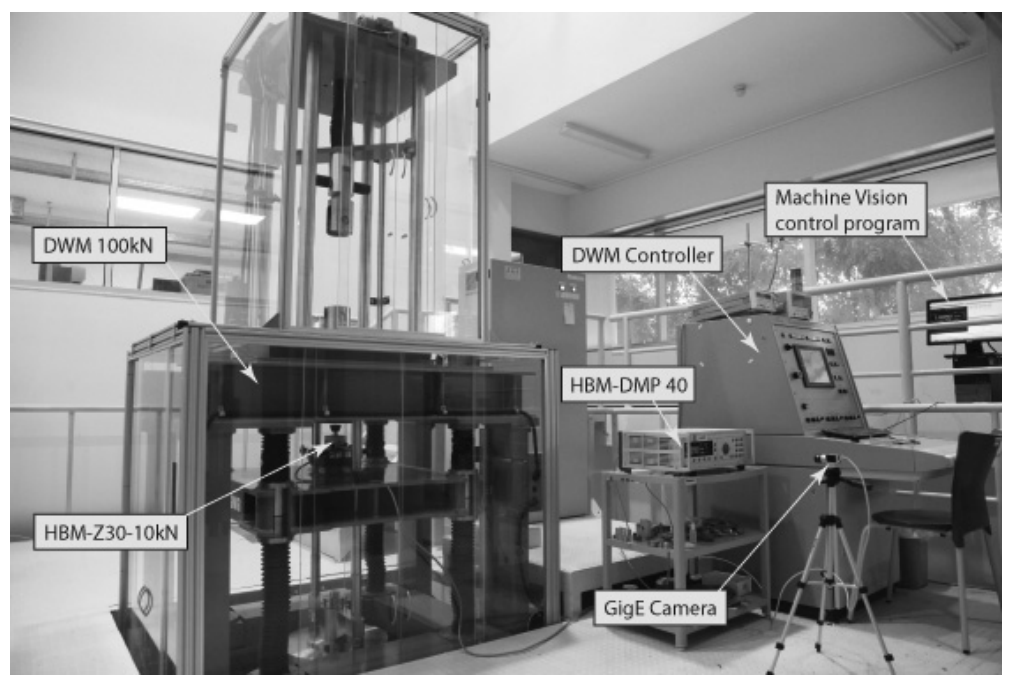

Fig.4. Experimental setup for verification of Machine Vision recording system. 
Figure 5 shows an example image of display of the amplifier (DMP40) along with the numerical data obtained using the developed program. The machine vision algorithm of the program was modified to be able to recognize the measurement image from the amplifier (DMP40). As seen in Fig. 5, the program was capable to translate the measurement image to numerical data correctly. Moreover, some other setting parameters of the amplifier during measurement can be observed in the image such as excitation voltage (5.0V) and selected channel (1.1).

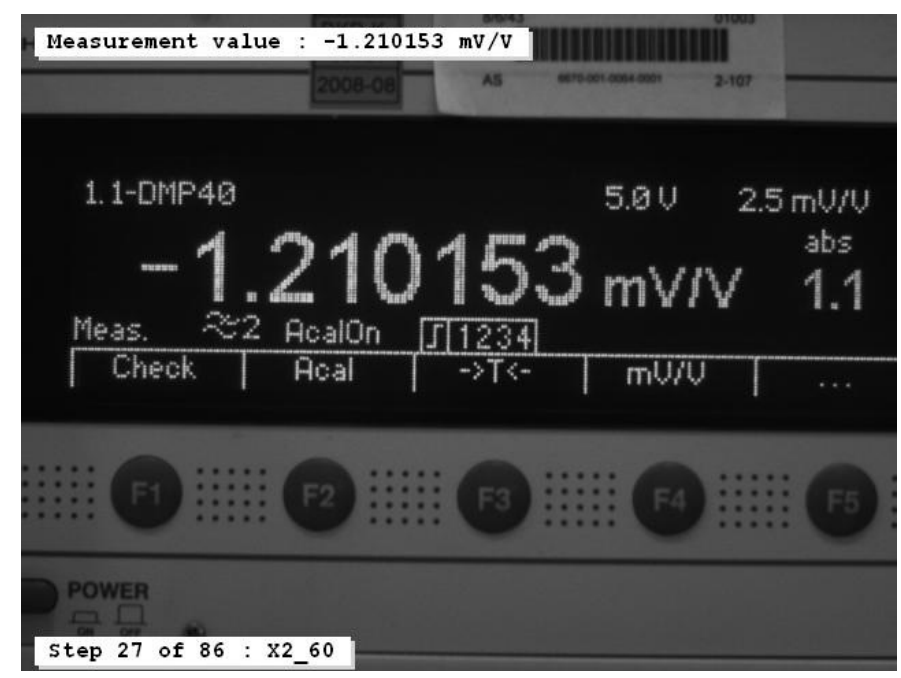

Fig.5. Measurement image and numerical data obtained using OCR technique.

As known that the OCR technique cannot guarantee $100 \%$ accuracy ${ }^{[7]}$, experiments were performed in 3 categories to evaluate the performance of this technique in force calibration.

$1^{\text {st }}$ - testing under dynamics condition. The controller of DWM was setup to apply force to load cell (HBM-Z30-10kN) from no load to maximum load and to no load $(0-10-0 \mathrm{kN})$. The machine vision system was used to record measurement data from the amplifier (HBM-DMP-40) during load change from 0 to $10 \mathrm{kN}$ and from 10 to $0 \mathrm{kN}$.

$2^{\text {nd }}$ - testing under statics condition. The controller of DWM was setup to apply increasing discrete forces series and decreasing discrete forces series to the load cell. In this case the developed system was used to record measurement data during stabilize time ( 30 - 45 seconds after loading) for each discrete force step.

$3^{\text {rd }}$ - full calibration process according to ISO376:2011. The DWM was used as a reference machine to calibrate the load cell (HBM-Z30-100kN). During calibration for each discrete force, the operator manually wrote calibration data into a calibration sheet and also pressed command button for the machine vision system to record the data. 


\section{Experimental Results}

Figure 6 shows an example of data obtained from the machine vision system recording under dynamics condition ( $1^{\text {st }}$ category). There are 112 points of experimental data in the figure. The red-circle marks (only 5 points) indicate that the raw image of that data having problem with overlapping number as seen in Fig. 7. These problems were from the update of the amplifier's display happen during the exposure time of the camera. The number of overlapping can be reduced by reducing the exposure time. For this study, the exposure time was intentionally set at about 2-scan time of the display. As seen in Fig. 6, only 5 from 112 points (\#13, \#58, \#66, \#82, \#94) having overlapping problem. All the points without overlapping were recognized by the system correctly. Averagely from dynamics experiments, the chance to have image with number overlapping is about $6 \%$.

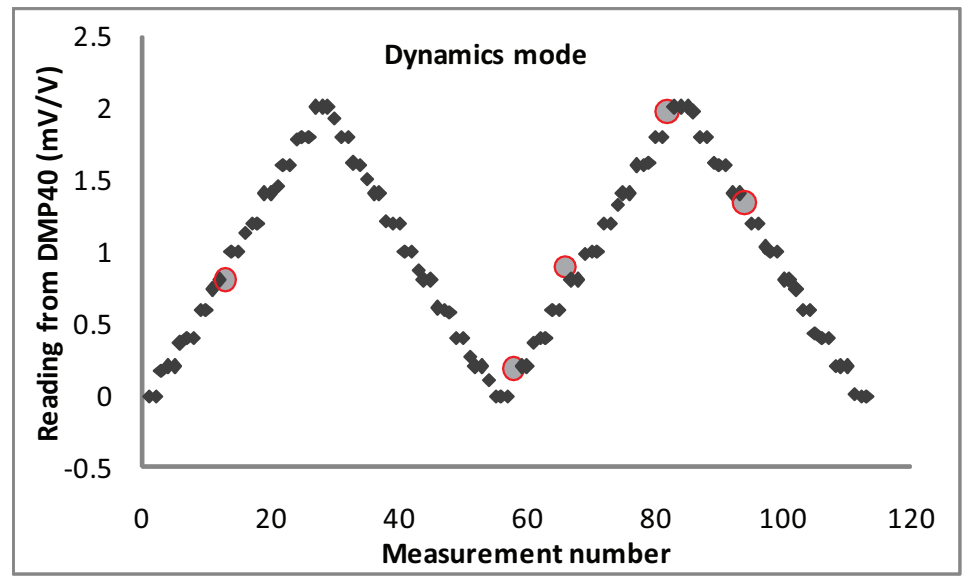

Fig.6. Example of measurement data obtained using OCR technique under dynamics mode.

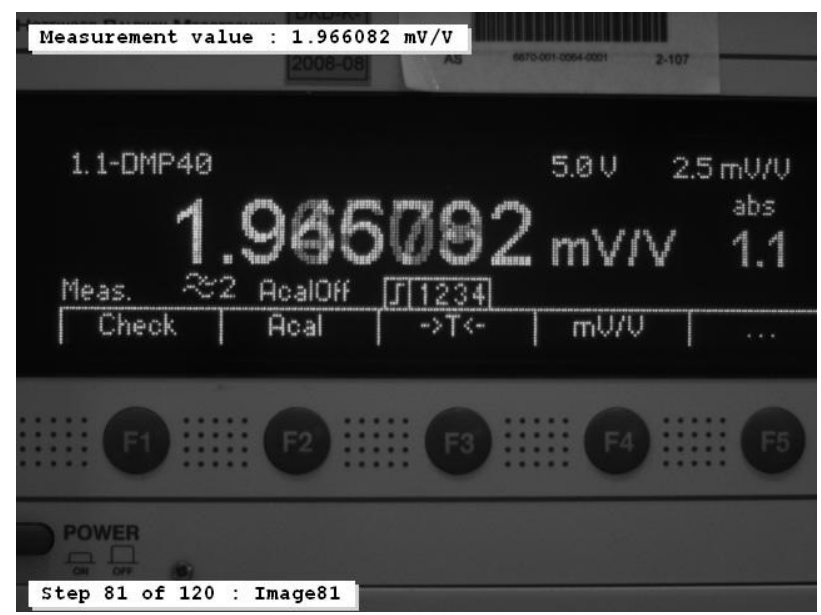

Fig.7. Example image with overlapping number. 
Figure 8 presents an example of data obtained from the machine vision system recording under statics condition ( $2^{\text {nd }}$ category). There are totally 105 points of experimental data in the figure. Only 1 point (\#99) had problem with overlapping data. All other points without overlapping were translated to numerical data correctly. Averagely from statics experiments, the chance to have images with number overlapping is about $2 \%$.

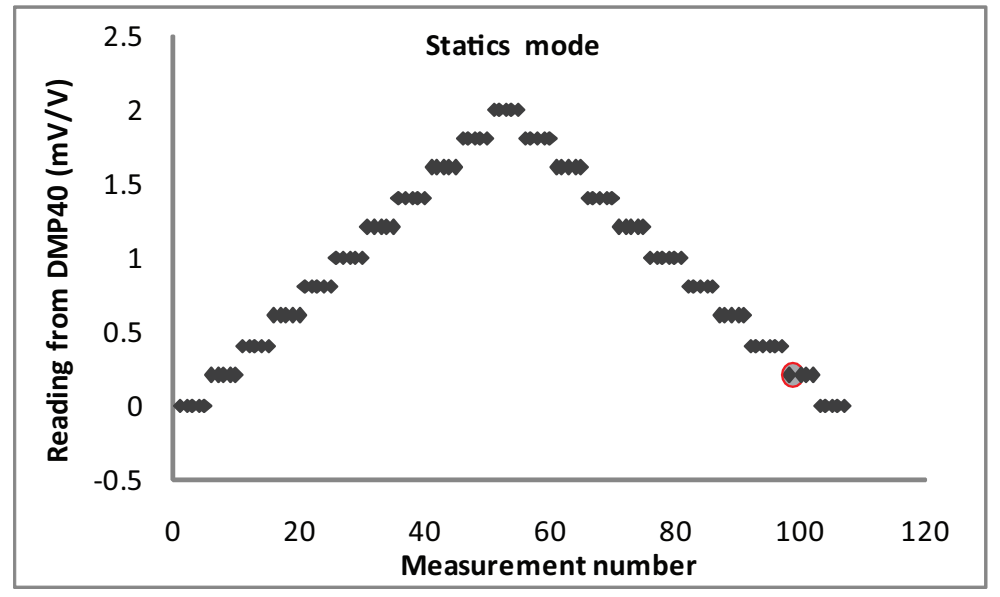

Fig.8. Example of measurement data obtained using OCR technique under statics mode.

Figure 9 shows an example of full calibration of load cell (HBM-Z30-10kN) in compression mode. There are 2 images with overlapping number. The system was programmed such that the operator can observe the overlapping problems and be able to press control button for retake the measurement image (dots next to red-circular marks). All the others points without overlapping were translated to numerical data correctly.

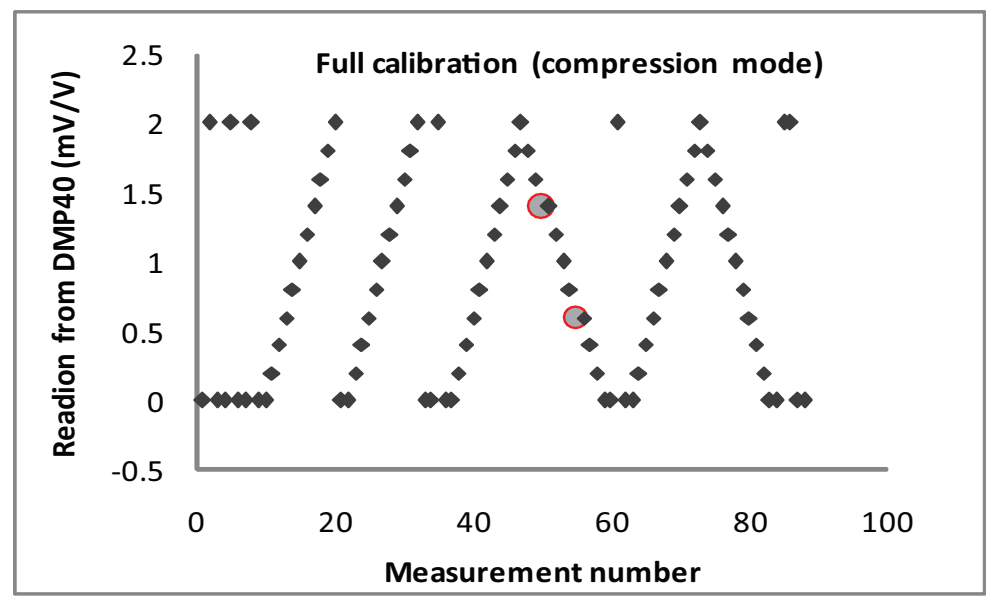

Fig.9. Example of full calibrations measurement data using OCR technique for compression mode. 
As seen in Fig. 10 (full calibration for tension mode), there was only one image with overlapping number. This problem (Fig. 11) was observed by the operator during calibration and the retaken image was shown in Fig. 12.

In force calibration, after application of each discrete force, it is recommended for waiting at least 30 second before reading the measurement data. Therefore, the statics condition strategy ( $2^{\text {nd }}$ category) could be applied. Chance of having overlapping number is $2 \%$ (Fig. 8). In case of taking 3 images for each calibration step, the chance to have all 3 images with overlapping number is only $0.0008 \%$ (calculated from $100 \times 0.02^{\wedge} 3$ ). It can be concluded that the successive rate of the system can increase by increasing number of repeated measurement.

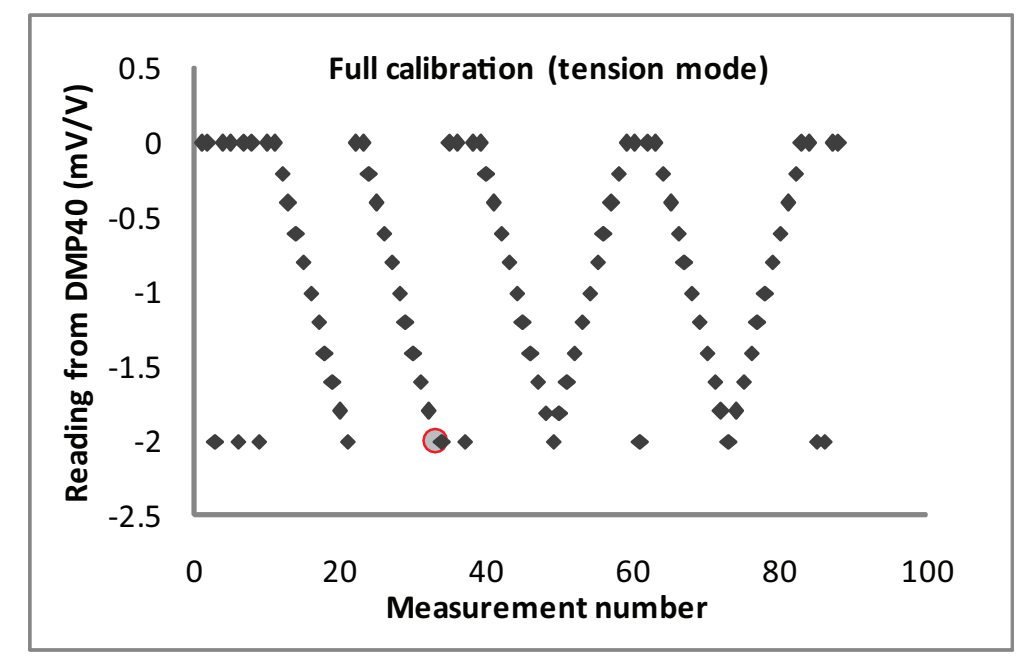

Fig.10. Example of full calibrations measurement data using OCR technique for tension mode.

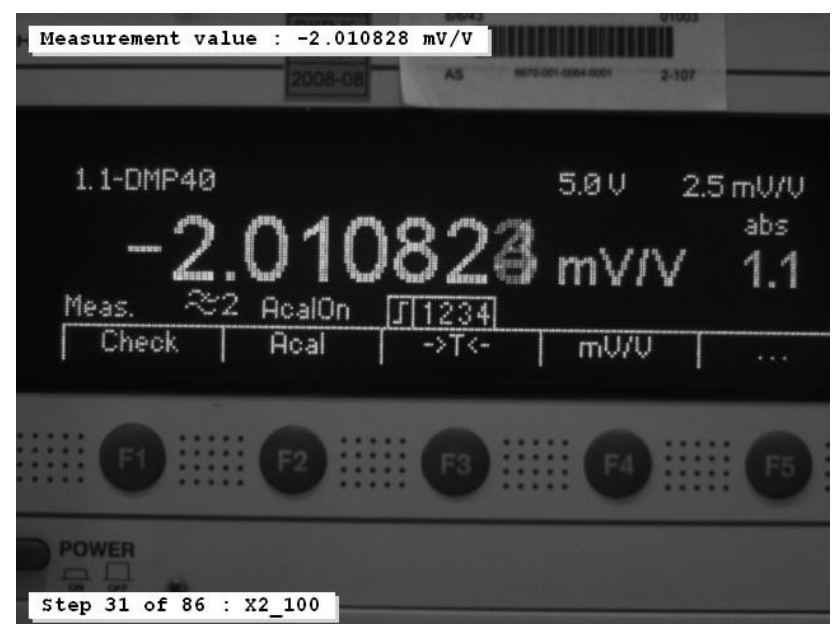

Fig.11. Image with number overlapping obtained during calibration. 


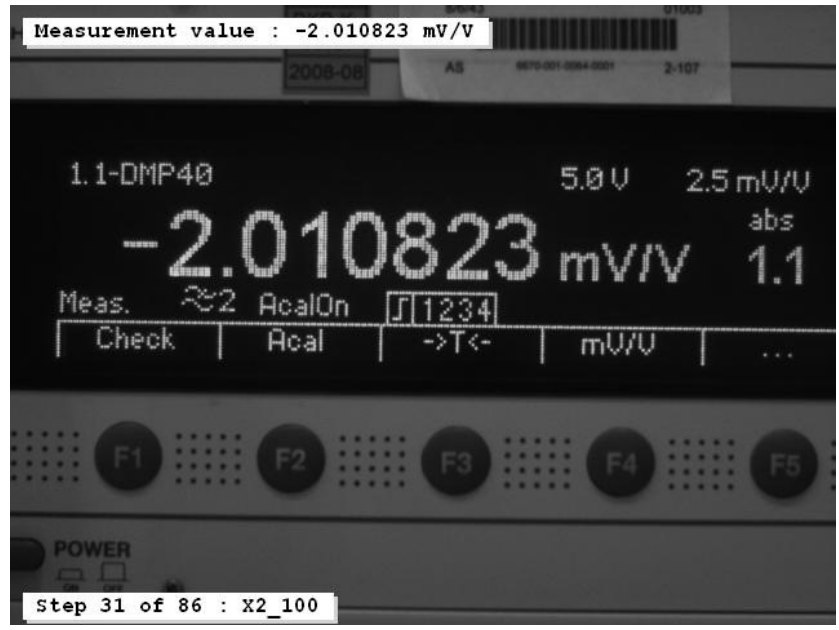

Fig.12. Retaken image after found problem with overlapping.

Table 1 presents an example of comparison of calibration result between manually recording and machine vision recording method. DWM-100kN was used as a standard to calibrate load cell (HBM-Z30-10kN) in compression mode. The interpolate value were calculate from least square fitting equation evaluated from the calibration data.

Table 1. Comparison of compression mode calibration results between manually recording method and machine vision recording method.

\begin{tabular}{|c|c|c|c|c|}
\hline \multirow{2}{*}{$\begin{array}{c}\text { Applied } \\
\begin{array}{c}\text { Force } \\
(\mathrm{kN})\end{array}\end{array}$} & \multicolumn{2}{|c|}{$\begin{array}{c}\text { Manually recording data } \\
\text { (Compression mode) }\end{array}$} & \multicolumn{2}{c|}{$\begin{array}{c}\text { Machine Vision recording data } \\
\text { (Compression mode) }\end{array}$} \\
\cline { 2 - 5 } & $\begin{array}{c}\text { Interpolate value } \\
(\mathrm{mV} / \mathrm{V})\end{array}$ & $\begin{array}{c}\text { Relative } \\
\text { Expanded } \\
\text { Uncertainty } \\
(\mathrm{k}=2)(\%)\end{array}$ & $\begin{array}{c}\text { Interpolate value } \\
(\mathrm{mV} / \mathrm{V})\end{array}$ & $\begin{array}{c}\text { Relative } \\
\text { Expanded } \\
\text { Uncertainty } \\
(\mathrm{k}=2)(\%)\end{array}$ \\
\hline 1.000 & 0.200122 & 0.0081 & 0.200122 & 0.0081 \\
\hline 2.000 & 0.400246 & 0.0059 & 0.400246 & 0.0059 \\
\hline 3.000 & 0.600373 & $\mathbf{0 . 0 0 5 2}$ & 0.600373 & $\mathbf{0 . 0 0 5 1}$ \\
\hline 4.000 & 0.800503 & 0.0042 & 0.800503 & 0.0042 \\
\hline 5.000 & 1.000636 & 0.0038 & 1.000636 & 0.0038 \\
\hline 6.000 & 1.200774 & 0.0035 & 1.200774 & 0.0035 \\
\hline 7.000 & 1.400916 & 0.0035 & 1.400916 & 0.0035 \\
\hline 8.000 & 1.601063 & 0.0035 & 1.601063 & 0.0035 \\
\hline 9.000 & 1.801215 & 0.0035 & 1.801215 & 0.0035 \\
\hline 10.000 & 2.001372 & 0.0035 & 2.001372 & 0.0035 \\
\hline
\end{tabular}


The relative expanded uncertainties were calculated from sources of calibration uncertainty recommended in ISO376:2011 (e.g. repeatability error, reproducibility error and reversibility error).

It is clearly seen from the table 1 that the results form machine vision recording method agree with the results from manually recording method. Based on experimental results, it can be concluded that the machine vision recording technique is appropriate for force calibration. However, the processing algorithm of program used in this study was valid only for recording measurement data from the amplifier (DMP40). In order to obtain numerical data from other models of amplifiers, modifications of the algorithm of the program to suite with each amplifier's display are necessary.

\section{Conclusions}

1. The developed machine vision system can be used for translation of measurement image to numerical data accurately.

2. The developed system help in reducing time of issuing calibration certificate because the operator do not have to enter the calibration data into calibration file manually.

3. With machine vision technique, it is possible to trace back to the original image obtained during calibration to make decision. Therefore, it can improve the quality assurance of calibration work.

4. From experiments, overlapping number of measurement image is a major source of problem with this technique. However, the chance of having image with overlapping can be reduce by reducing the camera exposure time and measuring data in statics condition.

5. From the experiment under dynamics condition, the chance of having image with overlapping is about $6 \%$. The chance of having overlapping is $2 \%$ in statics conditions. Multiple reading (such as 3 times) for each calibration step can reduce chance of failure to only $0.0008 \%$.

6. From the experiments, all images without overlapping problem were translated to numerical data with $100 \%$ accuracy.

7. The machine vision technique has excellent performance. Based on the experiment results, this technique is appropriate to be used in force calibration.

\section{Acknowledgments}

The authors are thankful to the National Institute of Metrology (Thailand) for the funding to perform this study. The authors would also like to thank Dr. Jirapong Kasivitamnuay and Mr. Somsak Charkkian for their valuable suggestions. 


\section{References}

1. ISO 376:2011(E), Metalic material - Calibration of force-proving instruments used for the verification of uniaxial testing machines (2011).

2. S. Wang, X. Yin, B. Ge, Y. Gao, H. Xie and L. Han, Machine vision for automated inspection of surgical instruments, Bioinformatics and Biomedical Engineering, ICBBE (2009).

3. T. H. Sun, C. C. Tseng and M. S. Chen, Electric contacts inspection using machine vision, Image and Vision Computing. 28, 890-901 (2010)

4. H. Y. T. Ngan, G. K. H. Pang and N. H. C. Yung, Automated fabric defect detection-A review, Image and Vision Computing. 29, 442-458 (2011)

5. I. Edinbarough, R. Balderas and S. Bose, A vision and robot based on-line inspection monitoring system for electronic manufacturing, Computer in Industry. 56, 986-996 (2005)

6. B.M. Sagar, G. Shobha and P. R. Kumar, OCR for printed Kannada text to machine editable format using database approach, WSEAS Transactions on Computers. 7(6), 766-769 (2008)

7. G. Andria, G. Cavone, L. Fabbiano, N. Giaquinto and M. Savino, Automatic Calibration System for Digital Instruments without Built-in Communication Interface, XIX IMEKO World Congress (2009)

8. S. E. Umbraugh, Digital Image Processing and Analysis: Human and Computer Vision Applications with CVIPtools, $2^{\text {nd }}$ edn. (CRC press 2011)

9. C. Steger, M. Ulrich and C. Wiedemann, Machine Vision Algorithms and Applications. (WILEY-VCH 2008) 